\title{
État des recherches en didactique du français
}

2 - Problèmes, objets et méthodes

\section{Anne Halté et André Petitjean}

\section{(2) OpenEdition}

Journals

Édition électronique

URL : http://journals.openedition.org/pratiques/1678

DOI : $10.4000 /$ pratiques. 1678

ISSN : 2425-2042

Éditeur

Centre de recherche sur les médiations (CREM)

Édition imprimée

Date de publication : 15 juin 2011

Pagination : 3-8

\section{Référence électronique}

Anne Halté et André Petitjean, «État des recherches en didactique du français », Pratiques [En ligne],

149-150 | 2011, mis en ligne le 13 juin 2014, consulté le 22 septembre 2020. URL : http://

journals.openedition.org/pratiques/1678; DOI : https://doi.org/10.4000/pratiques.1678 


\section{État des recherches en didactique du français. 2 - Problèmes, objets et méthodes}

Ce numéro de Pratiques constitue, à la suite du numéro 145/146 de juin 2010, le second volet de notre enquête consacrée à l'état du champ des recherches en didactique du français. Il y a un an, nous écrivions que le contexte politique et institutionnel n'était pas favorable aux recherches en didactique, évoquant notamment l'effet de l'intégration des IUFM aux universités et les réformes régressives de la formation des maîtres. Dans l'intervalle, le contexte n'a pas changé, il s'est même aggravé. Aujourd'hui, la mastérisation est effective, et force est de constater que les didactiques occupent bien souvent une place réduite dans les maquettes mises en place dans nombre d'universités. Il importe donc que Pratiques, qui a largement contribué à la genèse et au développement de la didactique du français, s'efforce d'établir une sorte de cartographie de ce champ de recherche.

Si le numéro 145/146 s'appuyait sur un certain nombre de thèses et HDR pour rendre compte de la diversité et de la richesse des recherches en didactique du français, ce second numéro complète le bilan par un certain nombre de synthèses susceptibles de rendre compte des orientations en cours. On le verra, il ne s'agit pas de prétendre à un panorama exhaustif de la discipline (ces deux numéros complètent d'autres parutions de Pratiques sur le même sujet) mais de mettre en relief certaines caractéristiques, tant en termes d'objets que de méthodes.

Le présent numéro se subdivise en trois sous-parties.

Dans une première partie, intitulée "Épistémologie », nous avons cherché à déterminer les caractéristiques essentielles de la discipline (finalités, objets, concepts, méthodes) ainsi que les types d'obstacles rencontrés (épistémologique, idéologique, corporatiste...) à la constitution et à l'institutionnalisation de la didactique du français comme discipline de recherche.

Concernant les problèmes, Bertrand Daunay et Yves Reuter se proposent d'analyser les difficultés institutionnelles et scientifiques rencontrées par la discipline. Les problèmes sont d'abord liés à la conjoncture politico-institutionnelle. La recherche en éducation, actuellement, est loin d'être une priorité ministérielle. Les auteurs montrent aussi combien certaines contraintes imposées à la recherche aujourd'hui (limitation dans la durée des thèses, importance accordée au quantitatif plus qu'au qualitatif...) ne sont pas des facteurs favorisants. Mais il existe aussi 
des difficultés institutionnelles inhérentes au champ même de la didactique du français. Cette dernière est inscrite dans divers cursus disciplinaires, ce qui ne facilite pas sa reconnaissance académique. A quoi s'ajoute le fait que ses objets d'étude relevant de compétences ordinaires du type «parler», «lire» ou "écrire», on dénie à la didactique du français toute nécessité scientifique d'une mise à distance problématisée de ces pratiques. Les auteurs insistent enfin, cette fois à l'intérieur même de la discipline, sur des points problématiques comme l'évaluation des travaux de recherche, des thèses notamment, et l'absence de mémoire des travaux antérieurs. A ces difficultés institutionnelles s'ajoutent les problèmes scientifiques, évoqués dans une dernière partie. La didactique du français, pour se constituer comme discipline autonome, a besoin de clarifier ses relations avec ses disciplines mères (notamment la linguistique et la littérature) et également avec ses disciplines contributoires (psychologie, sociologie..). Ce constat quelque peu déceptif n'empêche pas les deux auteurs de mesurer aussi les apports et l'inventivité d'une discipline en pleine évolution.

Au niveau des problèmes encore, Dan Savatovski, de son côté, s'intéresse aux formes de légitimation de la didactique du français (langue maternelle et langue étrangère). Après avoir listé un certain nombre d'indicateurs à l'aune desquels on peut mesurer la légitimité d'une discipline (types de supports éditoriaux, création d'emplois et profil des postes, plans de formation universitaires...), il précise qu'il limitera son analyse aux pratiques de la $7^{\mathrm{e}}$ section du CNU en matière de qualification des candidats dont les thèses relèvent de la didactique du français et des langues. Ce faisant, il montre que la didactique du français connaît des problèmes de frontières qui sont à la fois internes (ligne de partage entre le FLM, le FLE et le FLS) et externes (relations de proximité ou de distance avec des discipline connexes, qu'elles soient accréditées par la section CNU (psycholinguistique, sociolinguistique...) ou estimées hors champ (didactique de la littérature, sciences de l'éducation). Il apparaît ainsi que la didactique du français bénéficie d'une reconnaissance certaine au sein du CNU 07 depuis que la linguistique s'est ouverte aux sciences du langage. Ce qui n'empêche pas qu'elle souffre d'un partage de territoires sous-disciplinaires souvent discutables. A quoi s'ajoute le fait que les thèses qui s'affichent comme étant de « didactique » doivent proposer un cadre théorique qui convoque au moins un des paradigmes des sciences du langage. Ce qui se comprend mais peut parfois être handicapant pour une discipline de recherche souvent pluridisciplinaire, sauf à la concevoir comme de la linguistique appliquée.

Parmi les objets de recherche relativement nouveau pour la didactique du français, 1' " agir professoral » des enseignants, tel qu'il est observé et analysé à partir des interactions en classe, est au cœur de l'article de Francine Cicurel. Pour ce faire, cette dernière commence par rappeler et préciser les modes d'investigation possibles des interactions scolaires, à savoir : l'observation en classe, l'étude de la verbalisation des enseignants à propos de leur agir professoral, l'examen de documents qui précédent, accompagnent ou suivent leur action (cahier de préparation, journal de bord...). Dans un second temps, en forme de synthèse, Francine Cicurel rappelle les caractéristiques essentielles d'une interaction didactique (interaction planifiée et temporellement organisée, à visée cognitive et directive, comportant des jeux de langage particuliers entre des interactants qui occupent des places dissymétriques. Dans un troisième temps, à partir d'une étude de cas précise, Fancine Cicurel montre l'intérêt d'avoir recours aux discours des enseignants commentant leur propre pratique pour mieux appréhender l'ensemble des paramètres qui surdéterminent toute action professorale. 
Quant aux méthodes, elles sont abordées par Marie-Laure Elalouf à partir d'une réflexion consacrée aux corpus (contenus, constitutions, usages) dans les recherches en didactique du français. Son étude s'appuie sur la présentation de quatre thèses récentes qui ont en commun de contenir des corpus (souvent présentés en annexe) qui, au-delà de leur utilité pour les démonstrations propres à chacune des recherches, ont l'intérêt d'interroger la méthodologie même de la constitution d'un corpus. C'est ainsi que Marie-Laure Elalouf montre que ces corpus ont un double avantage. Intrinsèque, au sens où ils sont autant $d$ 'approches qualitatives qui donnent à voir la singularité des pratiques tant du côté enseignant que du côté élèves, tout en dégageant des éléments de typicité. Extrinsèque, dans la mesure où ces corpus constituent autant de banques de données qui, mises en réseau, permettent de passer d'études qualitatives à des études quantitatives, même si elles n'atteignent pas forcément la représentativité statistique. Dans une seconde partie de son article, Marie-Laure Elalouf envisage les différentes finalités que 1'on peut allouer à la constitution de corpus en didactique. Outre, bien sûr, la production de connaissances, ils peuvent jouer un rôle non négligeable à différents niveaux : matériaux disponibles dans la communauté scientifique, instruments de formation, et cela d'autant plus que le nouveau dispositif de formation des maîtres prive les futurs enseignants d'un contact réfléchi et problématisé avec la pratique des classes.

La seconde partie du numéro, intitulée "Phénomènes énonciatifs, sujet scolaire » a pour objectif de rendre compte du fait que l'élève, selon les disciplines et les activités, orales ou écrites, qui leur sont afférentes, est placé dans des situations particulières. Elles impliquent, outre des interlocuteurs, des pratiques interactionnelles et finalisées par l'intermédiaire de conduites cognitives d'enseignement-apprentissage.

C'est ainsi que Catherine Boré s'intéresse à l'élève en tant que sujet scripteur, et cela, à partir de l'analyse des brouillons. Dans un premier temps, elle s'efforce de caractériser, d'un point de vue énonciatif, le sujet scolaire, tel qu'il appartient à des scénographies particulières. Dans un second temps, elle mesure l'importance de la génétique textuelle consacrée aux brouillons pour la description des phénomènes liés à l'énonciation scripturale. En particulier le fait que la génétique a changé le statut du texte d'élève et aide à mieux comprendre (par l'élève comme par le maître) les opérations liées à la production des textes. Dans un troisième temps, Catherine Boré évalue ce que les théories de l'énonciation doivent à la perception dialogale de l'énonciation qui est celle de Benveniste et au dialogisme bakhtinien tels qu'ils ont été prolongés par le dialogisme interdiscursif d'AuthierRevuz. Ce cadre énonciatif posé, Catherine Boré apporte la preuve de leur fécondité en prenant pour exemple deux formes de dialogisme scolaire : le rapport du texte de l'élève à la consigne du maître, d'une part, la transcription du texte de l'élève par le maître, d'autre part.

Christine Deronne, de son côté, s'est penchée sur le mémoire professionnel, genre qui a été introduit dans la formation des maîtres en 1991-1992, au temps où l'on pensait qu'une année d'expérience professionnelle, étayée par une mise à distance réflexive, n'était pas inutile mais qui n'a pas survécu à la récente réforme de la formation des enseignants de 2010. Sur la base d'un corpus de mémoires professionnels du second degré en Lettres, Christine Deronne rappelle, dans la première partie de son article, les conditions institutionnelles de la production de ce type de mémoire ainsi que les normes scripturales afférentes à ce genre d'écrit. Il apparaît, dans la mesure où des fonctions diverses sont attribuées à ce genre scriptural (expériencielle, heuristique, réflexive, identitaire, certifiante) que l'identité du sujet 
scripteur est hétérogène et qu'il n'y a pas de consensus sur l'identification du mémoire professionnel en tant que mémoire de recherche. Dans une seconde partie, Christine Deronne rend compte de la variété et des variations des postures énonciatives des auteurs des mémoires professionnels. Elle le fait à partir de trois lieux d'observation : 1. l'auto-désignation du locuteur et la prise en charge énonciative de ses dire (tension entre implication et distanciation) ; 2. les modes de référenciation des objets de son discours selon qu'ils concernent les acteurs de la classe, les objets disciplinaires et didactiques spécifiques à l'enseignement du français, la nomination des actes de l'enseignant ; 3 . la dimension dialogique du mémoire (rapports interdiscursifs d'assujettissement ou de mise à distance des discours autres que sont les ouvrages de référence ou les textes officiels).

Martine Jaubert et Maryse Rebière, pour leur part, interrogent la place du langage dans les disciplines scolaires et sa prise en charge par la discipline du français. Il s'agit d'une problématique que partagent les didactiques. En effet, depuis une dizaine d'années, thèses, colloques, numéros de revue, traitent de cette question du langage (S.V.T., histoire, E.P.S...). Pour les auteures, la notion de position énonciative permet d'articuler langage et cognition et de reconfigurer l'enseignement du français. Il est du ressort de la didactique du français de réfléchir aux conditions permettant aux élèves de prendre conscience des variations langagières liées aux diverses disciplines. Cette didactique peut également fournir aux enseignants des outils pour faciliter les apprentissages dans les différentes disciplines. On sait que les élèves ont à construire des positions énonciatives propres à chacune d'elles et que la mise en cohérence et la stabilisation de ces différentes positions relèvent de l'enseignement du français. Après avoir évoqué les concepts émergents d'activité, de positionnement énonciatif, de variation langagière, de secondarisation, qui permettent de mieux traiter la question du langage dans les disciplines, les auteures donnent en conclusion des pistes de recherche pour de nouveaux objets pour la didactique du français. L'objectif est d'aider les élèves à maîtriser les variations énonciatives inhérentes aux diverses disciplines.

La troisième partie du numéro intitulée "Contenus et objets d'enseignement/apprentissage » a pour objectif, sous la forme de synthèses, de rendre compte des évolutions et des orientations qu'ont connues, dans leurs contenus et leurs méthodes, différents sous-domaines de la discipline, à savoir la linguistique "appliquée " aux textes d'élèves, la didactique du lexique, de l'oral et de l'orthographe, l'enseignement de la lecture littéraire.

L'article de Caroline Masseron qui ouvre cette troisième partie s'intéresse à la place que l'on peut accorder à la linguistique, selon la diversité des cadres théoriques qui constituent les Sciences du langage, à la fois pour élaborer de l'enseignable en matière de développement de la compétence scripturale des élèves et au service de l'analyse des productions d'élèves. Adoptant une perspective épistémologique et historique, Caroline Masseron retrace, durant la période qui va de 1975 à 2005 , les évolutions qui ont eu lieu d'un point de vue à la fois linguistique et didactique. Au niveau des théories de référence, différents paradigmes se sont imposés, à savoir : la « grammaire » de texte et les jugements de cohérence ; la psychogenèse des processus rédactionnels ; 1'énonciation, les types textuels, les mécanismes de cohésion des textes ; les genres d'écrits scolaires. Sur le plan didactique, les évolutions les plus marquantes, durant la même période, concernent l'attention portée à la genèse des textes et à la réécriture, le plaidoyer pour l'organisations des enseignements sous la forme de séquences et l'évaluation formative. Si l'on excepte les recherches en orthographe (analyse des procédures graphiques et 
typologies d'erreurs), les modèles linguistiques transposés ont été essentiellement des théories qui mettent l'accent sur la textualité et ses différents niveaux de structuration. Sans nier l'intérêt de ces travaux pour une didactique de l'écriture, Caroline Masseron regrette que l'analyse des productions écrites des élèves ait minoré les questions syntaxiques et milite pour une prise en compte des modèles macro-syntaxiques dont elle tente d'illustrer la validité.

Après avoir été reléguée longtemps au second plan des préoccupations didacticiennes, la didactique du lexique connaît aujourd'hui un regain d'intérêt. Francis Grossmann propose une synthèse fort utile des travaux récents qui lui sont consacrés. L'évolution des théories de référence en lexicologie et en acquisition ainsi que le renouveau des approches didactiques elles-mêmes, nécessite en effet un état des lieux. L'auteur montre d'abord que l'opposition entre apprentissage incident et apprentissage explicite n'a pas de raison d'être : les deux approches ne sont pas exclusives l'une de l'autre. Il revient sur des questions liées à l'enseignementapprentissage du lexique telles que la recherche du mot « juste» ou le vocabulaire de base. Sur ce dernier point, il préconise, plutôt que des listes de mots, la construction de lexiques selon les genres et les situations, intégrant la diversité et la variation. Dans la seconde partie de son article, Francis Grossmann expose les diverses voies qui se sont offertes à l'élaboration d'un enseignement structuré du lexique. A cette occasion, il se livre à une synthèse théorique d'approches variées, privilégiant la morphologie, l'analyse sémique, ou encore les polysèmes à l'étude des champs lexicaux et sémantiques ou des réseaux sémantiques. Il évoque aussi des approches plus « syntagmatiques », contribuant au décloisonnement du mot. Il associe à cette synthèse théorique une réflexion didactique sur les intérêts et les limites de chacune des approches. Enfin, il propose une réflexion sur l'intégration des apprentissages lexicaux aux activités de lecture, d'écriture et de production d'écrits. En conclusion, quelques pistes destinées à approfondir la réflexion sur la didactique du lexique sont autant d'incitations à poursuivre la recherche dans ce domaine.

C'est une mise en perspective très synthétique de la didactique de l'oral au cours des quarante années passées que propose Elisabeth Nonnon. Elle montre d'abord que les travaux sur l'oral n'occupent une place de choix dans les préoccupations didactiques que dans les situations de crise. Ceci s'est vérifié dans les années 70-80 et au tournant du XXI ${ }^{\mathrm{e}}$ siècle. Dans le premier cas, aux approches traitant l'échec scolaire en termes de handicap socio-linguistique, se sont opposées celles de divers chercheurs mettant en cause les travaux liant échec et déficit socio-culturel. Ces chercheurs ont impulsé une véritable réflexion, rappelée dans l'article, sur la problématique de l'oral. La seconde période, liée elle aussi à un malaise de l'école en rapport avec une crise sociale, a vu l'affinement des descriptions linguistiques de la langue parlée, la prise en compte des recherches en analyse conversationnelle et en sociolinguistique. Mais, pour Elisabeth Nonnon, dans la plupart de ces travaux, l'accent est mis plus sur le traitement des données orales que sur la didactique de l'oral, si l'on entend par là la recherche des formes de travail visant le développement des compétences orales de tous les élèves. L'auteure propose alors deux explications à cette difficulté de la didactique de l'oral à émerger. D'une part, elle analyse très finement les tensions liées à la question de la norme dans l'oral scolaire, d'autre part, elle souligne les différentes contraintes liées aux pratiques enseignantes relatives à l'oral : difficultés qui ne doivent pas empêcher d'espérer un retour de l'oral sur la scène didactique.

L'article de Catherine Brissaud s'ouvre sur le constat du décalage existant entre les recherches sur l'orthographe qui se sont multipliées depuis les années 70 et 
les pratiques de terrain, contraintes, entre autres, par les programmes et les évaluations.

S'interrogeant sur les apports des recherches menées depuis les années 70, Catherine Brissaud rappelle les travaux fondateurs qui ont contribué à affiner la description du système orthographique français. Elle montre aussi la meilleure connaissance que l'on a de l'acquisition de l'orthographe par les apprenants, grâce à la psychologie cognitive, à la linguistique et aux sciences de l'éducation.

Au début des années 2000, ces travaux se poursuivent dans une optique spécifiquement didactique, abordant des sujets peu traités ou polémiques, comme les difficultés orthographiques chez les étudiants et les adultes et les relations entre activités d'écriture et orthographe. Soucieuse de rendre compte des grands chantiers actuels en matière de didactique de l'orthographe, Catherine Brissaud en évoque trois : une réflexion sur l'inscription sociale des performances, la nécessité de conduire des études longitudinales d'envergure qui prennent en compte les contextes sociaux, un aménagement et du système d'écriture et des progressions en matière d'enseignement de l'orthographe à l'école. Après avoir examiné chacune de ces questions, elle conclut sur l'importance de la formation des enseignants et sur les liens entre recherche, formation et terrain en ce domaine.

Jean-Louis Dufays, pour sa part, constate et s'interroge sur le bien fondé du fait que l'approche par compétences a tendance aujourd'hui à se généraliser pour l'apprentissage de la lecture et de l'écriture, et ce, dans de nombreux pays. Dans un premier temps, adoptant un point de vue historique et épistémologique, il revient sur deux notions d'importance dans le cadre de la didactique de la littérature, à savoir : la lecture littéraire et le sujet lecteur. Il évoque d'abord l'évolution de la notion de lecture littéraire et rappelle l'intérêt de privilégier une tension dialectique entre lecture distanciée et lecture participative tout en alternant méthodiquement les deux postures. Il revient ensuite sur la notion de sujet-lecteur qui s'est développée dans les années 2000 et en montre les limites. Dans la seconde partie de son article, Jean-Louis Dufays témoigne de la complexité de l'approche par compétences. Il en analyse les répercussions sur les programmes belges de l'enseignement secondaire, tant dans le programme du réseau de la Communauté que dans celui du réseau catholique, et invite à réfléchir aux implications de cette approche sur la didactique de la littérature et sur les pratiques enseignantes de terrain. Le bilan critique proposé en fin d'article revient à la problématique initiale, et, à la lumière de l'exemple de l'enseignement en Belgique, pose une question fondamentale : l'objectif de l'école est-il de faire des élèves des interprètes performants ou des sujets dont l'expérience est enrichie et l'imaginaire nourri par leurs lectures?

Au total, avec ces deux numéros de Pratiques consacrés aux recherches en didactique du français, souhaitons que nous puissions un jour convaincre les autorités politiques (françaises) de son utilité sociale. En attendant, nous aurons tenté, sans prétendre nullement à l'exhaustivité, de mettre à la disposition des chercheurs et des formateurs, à la fois des résultats et des orientations nouvelles en voie d'expérimentation.

Anne Halté, André Petitjean 